\title{
Sampling of Elementary School Playground Equipment for Total Coliforms and E. coli
}

\author{
David Fowler ${ }^{1}$, Vanessa Karakilic ${ }^{2}$ \\ 1 Lead Authour, B. Tech Student, School of Health Sciences, British Columbia Institute of Technology, 3700 \\ Willingdon Ave. Burnaby, BC V5G 3H2 \\ 2 Supervisor, School of Health Sciences, British Columbia Institute of Technology, 3700 Willingdon Ave. Burnaby, \\ BC V5G 3 H2
}

\begin{abstract}
Background and Purpose: Outbreaks of enteric diseases in schools and daycares are common. It is possible that these outbreaks could be propagated via fomites in school settings, such as playground equipment that is not regularly cleaned. Studies thus far have provided conflicting results on the level of contamination present on fomites in the school setting. This project is intended to assess the level of microbial contamination present on elementary school playground surfaces as a result of hand contact from school children.

Methods: Two categories of elementary school playground equipment were sampled in this study; those that are likely to see regular hand contact from children and those that were not likely see hand regular hand contact from children. 30 surfaces of each category were swabbed and the media will be plated and incubated to enumerate total coliforms and E. coli.

Results: The mean number of total coliforms on high hand contact surfaces was 0.2333 $\mathrm{cfu} / 100 \mathrm{~cm}^{2}$, while the mean number of total coliforms on low hand contact surfaces was $0.2667 \mathrm{cfu} / 100 \mathrm{~cm}^{2}$. The t-test analysis of total coliform results produced a $\mathrm{p}$-value of 0.5566 . The mean number of $E$. coli on high hand contact surfaces was $1.1333 \mathrm{cfu} / 100 \mathrm{~cm}^{2}$, while the mean number of $E$. coli on low hand contact surfaces was $4.9000 \mathrm{cfu} / 100 \mathrm{~cm}^{2}$. The t-test analysis of $E$. coli results produced a p-value of 0.8019 .

Discussion: Neither results for total coliforms or $E$. coli indicated significantly different numbers on high or low hand contact surfaces. This indicates that these total coliform and E. coli may not be present as a result of hand contact.

Conclusion: These results do not support the deposition of coliforms or E. coli on playground equipment as a result of hand contact. However, there is still concern due to the number of samples positive for $E$. coli. These results suggest the need for practices such as regular hand washing in the school setting after using the playground, regular cleaning of playground materials and $\mathrm{EHO}$ inspections of school grounds.
\end{abstract}

Keywords: playgrounds, schools, surface sampling, sanitation, total coliforms, Escherichia coli, Abbotsford, BC 


\section{Introduction}

As an at risk population, young children are of particular concern when considering the threat of infectious disease outbreaks. Schools (Lee and Greig, 2010) and daycares (Goodgame, 2006) are common locations for outbreaks among children. The characteristics and behaviors of children such as diaper changing, poor bathroom hygiene, mouthing of toys, and inadequate hand washing can facilitate the spread of disease in these settings. Many enteric pathogens, such as viruses, can survive on hands and fomites for extended periods of time (Lee and Greig, 2010). As such, the level of contamination of objects such as school and daycare playground equipment and toys should be of concern to environmental health officers and staff wishing to reduce frequency and severity of outbreaks.

This project will investigate the level of microbial contamination introduced to school playground equipment by the hands of the children using it. This will be achieved by comparing the numbers of total coliforms and $E$. coli on surfaces of two categories of elementary school playground equipment; those that are likely to see regular hand contact from children and those that will not likely see hand regular hand contact from children. For example, handles going up to a slide would be considered to have regular hand contact, while the underside of the slide itself would be considered to have a low rate of hand contact. Three of each type of surface will be sampled at ten different schools. The selected surfaces will be sampled using 3M Quick Swabs, which will then be plated onto $3 \mathrm{M}$ Petrifilm ${ }^{\mathrm{TM}}$ E. coli/Coliform (EC) Count Plates. After incubation, the number of total coliforms and $E$. coli from each surface will be counted, and the average number of total coliforms and $E$. coli found on each surface will be calculated.

\section{Literature Review}

\section{Risk factors and history of daycare and school enteric disease outbreaks}

In order to understand how outbreaks occur in the school and daycare setting it is important to look at the information available on previous outbreaks. The level of contamination present in the daycare setting has been studied significantly, though with inconsistent results. Ibfelt (2015), found very few surface samples positive for $E$. coli in their investigation. This seems to contrast results typical of many studies previous to Ibfelt (2015) which the authors referenced, some of which found $20-50 \%$ of samples from toys or other fomites to be positive for $E$. coli (Ibfelt, 2015). There is however, strong evidence to suggest that daycares and schools are subject to high rates of gastrointestinal disease outbreaks (Goodgame, 2010; Lee and Greig, 2010). Enserink et al (2015) studied the risk factors in daycare settings for gastroenteritis. The following factors were identified to be associated with higher risk of gastroenteritis cases in the facilities: crowding, diaper change areas, sandpits, paddling pools, cleaning potties in sinks, cleaning vomit without disinfectant, mixing staff between child groups and staff with multiple duties (Enserink et al, 2015). In contrast, the following protective factors were identified: disinfecting fomites with chlorine, cleaning vomit with paper towel and disinfectant, daily cleaning of blankets and toys, and exclusion of sick staff or children (Enserink et al, 2015). From this study, it seems that sanitation practices are 
key to reducing the frequency of these illnesses.

Lee and Greig (2010) reviewed 121 documented gastrointestinal outbreaks in the Canadian and American school setting between the years of 1998 to 2008 . Sources of outbreaks identified included direct and indirect person to person transmission, contaminated food from lunch programs, food handlers with poor hand hygiene, sick workers, and animal contact (Lee and Greig, 2010). The causative agents of these outbreaks were $51 \%$ bacterial, $40 \%$ viral and $7 \%$ protozoan (Lee and Greig, 2010).

A similar study was done by the same authors, Greig and Lee (2008), this time in the daycare setting. This study reviewed 75 studies in which 1806 children were reported ill, 104 of which were hospitalized (Greig and Lee, 2008).

The conclusions and recommendations from these two studies had a great deal in common. The most common measures to address the outbreaks included alerting health authorities, treating ill, increased hand washing and increased vigilance during food preparation (Lee and Greig, 2010). Particular emphasis was given in both studies on the prevention of person-toperson transmission through enhanced hand washing and the use of hand sanitizers and wipes. The lack of proper hand hygiene in young children is common and has been supported by numerous studies. Lee and Greig (2010) cite surveys indicating that amongst 4 schools in Pennsylvania, $71 \%$ of seventh graders seldom washed their hands before eating at the school cafeteria. The results of these studies and knowledge of childhood behavior suggest that there is good reason to investigate the level of contamination present on environmental surfaces in schools and daycares.

\section{Pathogens commonly associated with school and daycare outbreaks}

As noted by Lee and Greig (2010), most gastrointestinal outbreaks in these settings are caused by either viral or bacterial enteric pathogens. Enteric pathogens are spread via the fecal oral route (Public Health Agency of Canada [PHAC], 2015; PHAC, 2014), and the confined environments of schools or daycares is ideal for direct or indirect transmission of these diseases (Lee and Greig, 2010). Some classic examples of these pathogens include norovirus and E. coli O157:H7.

Data on the prevalence of norovirus is limited due to the rapid onset and short duration of the illness (Goodgame, 2006). However, it is estimated to cause 60 to $95 \%$ of episodes of acute diarrhea and/or vomiting not caused by bacteria (Goodgame, 2006). Fomite transmission of this virus is typical (Goodgame, 2006), and is facilitated by its long survival times on environmental surfaces and low infectious dose. The Public Health Agency of Canada (2015) indicates an infectious dose of less than 10 virions. Kim et al (2014) studied the survival time of norovirus on different types of surfaces. They noted that steel had the greatest reduction in infective virions, while wood had the least reduction (Kim et al, 2014). However, all six types of surfaces tested retained infective virions after 28 days (Kim et al, 2014), and other studies have shown virions to remain infective on steel surfaces for even longer.

As an example of a bacterial pathogen, E. coli 0157 shares many similarities to norovirus in terms of transmission potential. The Public Health Agency of Canada (2014) indicates the infectious dose to be as low as 10 organisms 
ingested. A study done by Wilks et al (2005), investigated survival times of $E$. coli 0157 on different types of metallic surfaces. Survival times of over 28 days were found at refrigeration and room temperatures on stainless steel, though they did undergo a 5 log reduction in viable bacterial counts in the first 2 days (Wilks et al, 2005). It was interesting to note the antibacterial properties of copper and copper alloys, which effectively reduced viable bacterial cells to 0 after only 2 hours (Wilks et al, 2005).

The results of these studies further support the investigation of environmental surfaces for levels of contamination in the school and daycare setting, by simply supporting the extended survival times of pathogens on fomite surfaces.

\section{Other Pathogens identified on Playground surfaces}

French et al (2009), investigated the prevalence of Campylobacter jejuni in isolates of bird fecal matter taken from outdoor playgrounds. They demonstrated that these isolates did indeed contain Campylobacter and were frequently strains capable of causing disease in humans (French et al, 2009).

Furthermore, the material surrounding playgrounds has been shown to harbor pathogens. For example, Staff et al (2012) reported an outbreak of Salmonellosis in Australia that was traced back to ingestion of contaminated playground sand. The source of this pathogen was suggested to be fecal mater introduced to the sand by local wildlife. Interestingly, the researchers noted that the bacterium was capable of surviving in the playground sand for up to 9 months. This not only demonstrates the potential for playgrounds to act as pathogen reservoirs, but also reinforces the need to carefully consider the materials used in constructing the playgrounds themselves (Staff et al, 2012).

\section{Surface sampling and indicator organisms}

In order to investigate the contamination levels of environmental surfaces, an appropriate sampling technique must be used to isolate and quantify the target organism. While sampling for the pathogens themselves would be ideal, this is typically not practical when surface sampling. Pathogens are typically found in numbers low enough that they are difficult to detect, and viral pathogens can not be cultured on media alone (Montville, 2008). As such, indicator organisms are used to indicate the potential presence of the pathogenic organisms (Montville, 2008). In the case of enteric pathogens, fecal indicators are typically used (Montville, 2008). Historically, fecal coliforms have been used for this purpose, but current practice favors the use of $E$. coli as a fecal indicator (Greig and Lee, 2008).

The BCCDC (2014), does provide parameters regarding acceptable levels of fecal coliforms or $E$. coli in ready to eat foods. However, these parameters do not apply to environmental surfaces. Instead, the BCCDC (2014) only provides guidelines on the acceptable level of aerobic colony counts (ACC) on food preparation surfaces for the purposes of educating restaurant operators on proper sanitation practices.

\section{Alternative Culturing Methods}

Traditional culturing methods of indicator organisms can be cumbersome and time consuming. Methods to streamline the procedures have been developed to simplify the process and make it less labor intensive. The following studies evaluate 
some of the traditional methods and alternative culturing methods.

Jasson et al (2010) have provided a review of some surface sampling methods. They note that alternative methods require $3^{\text {rd }}$ party validation to be accepted by authorities. Aside from the time and labour, they note that traditional methods are limited to minimum counts of $4 \mathrm{cfu} / \mathrm{ml}$ for liquids and $40 \mathrm{cfu} / \mathrm{ml}$ for solids. Below this, MPN (most probable number) techniques must be used, which are also time a labor intensive (Jasson et al, 2010). $3 \mathrm{M}$ Petrifilm ${ }^{\mathrm{TM}}$ are discussed as an alternative method that is much less labor intensive, and the Petrifilm ${ }^{\mathrm{TM}}$ themselves are flexible and occupy less space (Jasson et al, 2010).

Claro et al (2014) investigated the limits of detection using swabs, contact plates and petrifilms and determined that Petrifilms ${ }^{\mathrm{TM}}$ were the best method for recovery of MRSA and a particular strain of E. coli (Claro et al, 2014).

Saab et al (2013) also supported the Petrifilm ${ }^{\mathrm{TM}}$ in their studies for recovering $E$. coli from cheese samples. They reported detection of $22 \times 10^{6} \mathrm{cfu} / \mathrm{g}$ for Petrifilm ${ }^{\mathrm{TM}}$ samples as compared to $12 \times 10^{5} \mathrm{cfu} / \mathrm{g}$ from conventional plating techniques (Saab et al, 2013).

\section{Alternative Rapid Methods}

While the enumeration of indicator organisms such as $E$. coli is a widely accepted practice for evaluating the presence of fecal material (BCCDC, 2014), there are alternative to traditional culturing methods that can yield much faster results. For example, ATP bioluminescence is a technique that can be used to evaluate overall hygiene levels on a surface (Montville, 2008). While traditional culturing methods may require growth of samples on solid or liquid media for 2 to 7 days, ATP bioluminescence produces results within 5 minutes (Chollet and Ribault, 2012). This technique makes use of a light producing reaction, catalyzed by the enzyme known as luciferase (Chollet and Ribault, 2012). The enzyme requires ATP to function (Chollet and Ribault, 2012). Thus, the amount of light produced is dependent on the amount of ATP present on the sampled surface (Chollet and Ribault, 2012). ATP on the surface is proportional to the degree of contamination with microbial and organic matter (Chollet and Ribault, 2012). The major drawbacks of this technique are its lack of specificity (Montville, 2008) and that the Relative Light Units measured are specific for each device, and thus require calibration against total colony counts to be used as anything more than a general indicator of hygiene (Mulvey et al, 2011). That said, studies have been done to attempt to develop a benchmark for acceptable ATP bioluminesence readings. Mulvey et al (2011) used the Hygenia ATP bioluminescence system to determine RLU levels that correlated with bacterial aerobic colony counts. In particular, they determined that less than $250 \mathrm{RLU}$ correlated to surfaces with little organic material while greater than 1000 RLU indicated poorly cleaned surfaces (Mulvey et al, 2011).

\section{Strengths and limitations of the literature review}

This literature review focused on sampling techniques that were frequently referenced in the literature. There are many other alternative sampling techniques that did not enter discussion in the review, however, those most appropriate to sampling irregularly shaped surfaces, such as on playgrounds, have been discussed. 
Gaps in research, policy and knowledge:

While studies were found on outbreaks in the school setting, there were few studies to be found on the microbial contamination present in the school setting. In contrast, a great deal of research has been focused on daycares and the presence of pathogens in this setting.

There are a lack of guidelines or legislation that refer to acceptable levels of contamination on environmental surfaces outside of food establishments. Thus, this project will seek to compare different surfaces as opposed to making comparisons to a pre-determined standard.

\section{Literature Review Conclusions:}

Outbreaks of enteric disease pose a real and ongoing problem in the daycare and school environment. The susceptibility of young children to disease demands that environmental health professionals take special care to determine how disease outbreaks are spread and what they can do to prevent or mitigate them. Multiple sources indicate that both hand hygiene and contaminated fomites play an important role in permitting the transmission of these diseases from person to person (Greig and Lee, 2008; Lee and Greig, 2010). This suggests that further investigation into the level of contamination on fomites such as toys and play equipment should be done, particularly in the school setting where much of this information is lacking.

There are many options when it comes to surface sampling. Culturing methods that enumerate a particular indicator organism are widely accepted. $E$. coli is the most widely accepted indicator of fecal contamination, and thus indicates the potential presence of enteric pathogens (Montville, 2008).

Rapid methods could also be utilized to provide a general knowledge of the sanitation levels of the surfaces in question. ATP bioluminescence has gained prominence as a rapid method (Chollet and Ribault, 2012). It provides rapid results about the level of microbial or organic matter present, but specific RLU measurements may be specific to a particular brand of measuring devices (Mulvey et al, 2011).

Information on the contamination levels present in the school and daycare settings will help public health professionals determine what needs to be done to prevent future outbreaks. Cleaning practices, hand washing promotion and even the types of materials used for playground construction could all be important considerations if surface contamination continues to prove to be a problem in the school and daycare setting. As results of this literature review suggest that $E$. coli is the standard for indicating fecal contamination and the presence for human pathogens, this project will utilize methods to enumerate the number of $E$. coli on playground surfaces. In particular, petrifilms have been emphasized for their reliable and convenient use in recovering $E$. coli from environmental surfaces (Saab et al, 2013). As such, this project will make use of $3 \mathrm{M}$ Petrifilm ${ }^{\mathrm{TM}}$ E. coli/Coliform (EC) Count Plates. The overall objective of this project, will be to compare E. coli (and total coliform) numbers on playground surfaces that see regular hand contact to those that do not in order to determine just how much contamination can be attributed to hand contact on these surfaces. 


\section{Methods and Materials}

The purpose of this study is to assess the contamination levels on high hand traffic surfaces on school playgrounds. In order to do so, two different categories of surfaces were sampled at each playground. High hand traffic surfaces were selected based on having regular contact with the hands of children using the playground. For the purposes of this experiment, monkey bars were consistently selected for this surface category. The second surface category of surfaces were selected based on their having lower rates of contact by the children using them. For this study, low hand traffic surfaces sampled included the underside of slides or, if slides were unavailable, out of the way structural posts. These surfaces were swabbed and the swab media was used to enumerate two types of indicator organisms; total coliforms and $E$. coli. Total coliforms are an indicator of general hygiene, while $E$. coli are an indicator of fecal contamination and suggests the potential for the presence of human pathogens (Montville, 2008). The hypotheses for this study are as follows:

Ho(1): There will be no significant difference between the mean number of total coliforms on high hand traffic playground surfaces and on low hand traffic playground surfaces.

$\mathrm{Ha}(1)$ : There will be a significant difference between the mean number of total coliforms on high hand traffic playground surfaces and low hand traffic playground surfaces.
Ho(2): There will be no significant difference between the mean number of $E$. coli on high hand traffic playground surfaces and on low hand traffic playground surfaces.

$\mathrm{Ha}(2):$ There will be a significant difference between the mean number of $E$. coli on high hand traffic playground surfaces and low hand traffic playground surfaces.

\section{Materials}

$3 \mathrm{M}$ Petrifilm ${ }^{\mathrm{TM}}$ EC plates allow the enumeration of total coliforms and $E$. coli contained in $1 \mathrm{ml}$ of sample media (3M, 2014). These plates contain Violet Red Bile (VRB) nutrients, a cold-water-soluble gelling agent, an indicator of glucuronidase activity and an indicator to allow colony enumeration (3M, 2014). These plates function on the principle that most $E$. coli produce beta-glucuronidase, which when combined with the indicator on the plates, shows up as a blue colony (3M, 2014). Coliforms, including $E$. coli, also produce acid and a gas during lactose fermentation (3M, 2014). The acid causes coliform colonies to appear red (due to the $\mathrm{pH}$ indicator), while the gas is trapped by the film over top of the plate, appearing as a bubble (3M, 2014). Thus, coliforms show up as red colonies with a gas bubble, while E. coli appear as blue colonies with a gas bubble (3M, 2014).

Surface sampling was done using $3 \mathrm{M}$ Quick Swabs. This swab can be used on wet or dry surfaces and can be used in conjunction with any $3 \mathrm{M}$ Petrifilm ${ }^{\mathrm{TM}}$ Plate. The swab container itself contains $1 \mathrm{ml}$ of sodium bisulfite-free letheen broth, to which the recovered bacteria are deposited. From here, the $1 \mathrm{ml}$ of media can be transferred to the $3 \mathrm{M}$ Petrifilm ${ }^{\mathrm{TM}}$ Plate (3M, 2014). 
A total of 60 playground surfaces were sampled during this study. Each surface sampled required 1 Quick Swab and $13 \mathrm{M}$ Petrifilm ${ }^{\mathrm{TM}}$ E. coli/Coliform (EC) Count Plate. One additional swab and plate will be used as a control from an laboratory surface. Thus, a total of 61 swabs and Petrifilm ${ }^{\mathrm{TM}}$ plates were used. Swabbed surfaces were cleaned with pre-packaged alcohol swabs to prevent encouraging bacterial growth on the sampled playgrounds. Pre-cut paper templates were used to ensure a regular surface area of $100 \mathrm{~cm}^{2}$ was sampled on each surface. Sampled swabs were kept cold by holding them in a cooler containing ice and test tube racks to prevent loss of sample.

When plating the sample media, a plastic spreader was used to distribute the sample over the Petrifilm ${ }^{\mathrm{TM}}$ plate and the plates were incubated at $35^{\circ} \mathrm{C}$ in an incubator.

\section{Procedures}

\section{Surface Sampling with 3M Quick Swabs}

Sampling of all surfaces was conducted from 4:00pm to 8:00pm on a Tuesday afternoon. Weather leading up to the sampling had been sunny and clear, and sampling was done afterschool on Tuesday to allow for recent use of the playgrounds sampled. 15 elementary schools in the Abbotsford school district were selected, ranging in location from East to West Abbotsford. At each school, two high contact and two low contact surfaces were sampled.

Prior to sampling, each swab was labelled by number, and a key was recorded matching the number to surface type and location as sampling proceeded. During the sampling process, swabs and swab media were held in a cooler with ice. A $100 \mathrm{~cm}^{2}$ area of the experimental surface was selected for sampling using the paper templates. Procedures for swabbing the surfaces were conducted according to the 3M Quick Swab Product Instructions (3M, 2014). The bulb containing the media was broken and the bulb was massaged to release the media to the swab head. The swab was removed from the tube containing the media. The swab was held at a 30-degree angle to the sampled surface and rubbed back and forth, side to side, along the length of the desired surface area. This process was repeated with strokes moving 90 degrees to the original stroke direction, and then repeated a third time moving at a 45-degree angle to the original stroke direction. The swab was returned to the broth filled tube. The surface sampled was cleaned with alcohol and wipes and the swab tube and media were returned to the cooler. Swab samples were held in a fridge at $4^{\circ} \mathrm{C}$ overnight, and transported on ice to the lab the next day, where they were plated (from 2:30-3:30pm) 23 hours after sampling.

\section{M Petrifilm ${ }^{\mathrm{TM}}$ EC/Coliform Plating Procedures}

Plating was done according to the $3 \mathrm{M}$ Petrifilm ${ }^{\mathrm{TM}} \mathrm{EC} /$ Coliform Product Instructions (3M, 2011). The entire content of the Quick Swab Sample broth $(1 \mathrm{~mL})$ was poured onto the center of the plate while holding the plastic cover away from the plate. The plastic cover was lowered, and a flat spreader was used to carefully spread the sample beneath the plate cover. The plates were left for 1 minute to allow the gel to set. The plates were placed in the incubator with the clear side up. Plates were incubated at $35^{\circ} \mathrm{C}$ for 24 hours prior to coliform enumeration and 48 hours prior to E. coli enumeration. Coliforms were identified as red colonies with a gas bubble, 
while $E$. coli colonies were blue with a gas bubble (3M, 2014).

\section{Inclusion and Exclusion Criteria}

Fifteen elementary schools were randomly selected from the Abbotsford School district for sampling. Only playgrounds from elementary schools were sampled.

High and low hand traffic surfaces were defined based on the likely use of the surface. High contact surfaces on the playground were limited to monkey bars for consistency. For low contact surfaces, the underside of slides were prioritized, while structural posts in out of the way locations were sampled in the case of a lack of available slides.

\section{Ethical Considerations}

As this study did not involve human or animal subjects, or a survey, there were few ethical considerations necessary. However, it was important to adequately clean the sampled playground surfaces after sampling, as these procedures introduced media designed to allow the growth of many types of bacteria. This was done using ethanol wipes.

\section{Statistical Analysis}

The data produced by this study was in the form of colony forming units (cfu) for both the total coliforms and $E$. coli counts. It was assumed that each cfu represented 1 vegetative cell. This data was a discrete, numerical data. The average total coliform and $E$. coli counts for each type of surface were represented by the arithmetic mean. The mean is commonly used to represent normally distributed numerical data, such as what was expected to be produced in this study (Heacock \& Karakilic, 2015).
Statistical analysis was done using SAS software (SAS University Edition, 2015). SAS was used to run an independent sample, upper one tailed t-test. The upper one tailed t-test was used for determining if the means of two data sets are significantly different, when one mean was expected to be higher than the other (Heacock \& Karakilic, 2015). In the case of this study, the Ha's stated that the coliform and E. coli counts were expected to be higher on high hand traffic surfaces as compared to low hand traffic surfaces. It should be noted that there are three assumptions that must be made about the data sets when using this type analysis. First of all, it was assumed that the data set for each group is normally distributed (Heacock \& Karakilic, 2015). If this were not the case, then it would be necessary to conduct a nonparametric test (the Wilcoxon Rank Sum Test) (Heacock \& Karakilic, 2015). SAS is capable of determining if data sets are normally distributed (Heacock \& Karakilic, 2015). Secondly, the standard deviations of the two data sets were assumed to be equal (Heacock \& Karakilic, 2015). As the number of samples taken for each surface type were equal, their standard deviations should be equal as well (Heacock \& Karakilic, 2015). Thirdly, the data observed in each group must be independent from the values in the other group (Heacock \& Karakilic, 2015). This should have been true, as it was not anticipated that the level of contamination on one surface type would be directly related to the level of contamination on the other surface type (Heacock \& Karakilic, 2015).

As the $3 \mathrm{M}$ Petrifilms ${ }^{\mathrm{TM}}$ provided data on both the total coliform and E. coli counts for the sampled surfaces, separate but identical analyses were done for the total coliforms and E. coli counts. 


\section{Results}

Total Coliform counts for the high contact playground surfaces and low contact surfaces were found to be normally distributed by the SAS analysis. The calculated $p$ values for the normality tests were $<0.05$, indicating both data sets were normally distributed (Heacock \& Karakilic, 2015). Thus, the Parametric t-test results were used.

The mean Total Coliforms count for high hand contact surfaces was calculated to be $0.2333 \mathrm{cfu} / 100 \mathrm{~cm}^{2}$ (with a maximum of 6 , minimum of 0 and standard deviation of 1.1043). The mean Total Coliforms for low hand contact surfaces was 0.2667 $\mathrm{cfu} / 100 \mathrm{~cm}^{2}$ (with a maximum of 2 , minimum of 0 and standard deviation of 0.6397). This analysis used an upper tailed, one-way t-test. The calculated $p$ values for equal (0.5566) and unequal (0.5566) variance were greater than 0.05 . This indicated that the average Total Coliform count for high hand contact surfaces was not significantly different than the average Total Coliform count for low hand contact surfaces. Thus, it is not possible to reject the Ho(1) (Heacock \& Karakilic, 2015).

E. coli counts for high contact playground surfaces and low contact playground surfaces were found to be normally distributed by the SAS analysis. The calculated $p$ values for normality tests were less than 0.05 , indicating both data sets were normally distributed (Heacock \& Karakilic, 2015). Thus, the Parametric t-test results were used.

The mean $E$. coli count for high hand contact surfaces was calculated to be $1.1333 \mathrm{cfu} / 100 \mathrm{~cm}^{2}$ (with a maximum of 15 , minimum of 0 and standard deviation of 2.9680). The mean E. coli for low hand contact surfaces was $4.9000 \mathrm{cfu} / 100 \mathrm{~cm}^{2}$

(with a maximum of 131, minimum of 0 and standard deviation of 23.9530). This analysis used an upper tailed, one-way ttest. The calculated $p$ values for equal (0.8019) and unequal (0.8003) variance are greater than 0.05 . This indicated that the average $E$. coli count for high hand contact surfaces was not significantly different than the average $E$. coli count for low hand contact surfaces. Thus, it is not possible to reject the $\mathrm{Ho}(2)$ (Heacock \& Karakilic, 2015).

\section{Discussion}

The results of this study failed to reject the null hypotheses for total coliforms and for E. coli. This means that for high and low hand contact surfaces on elementary school playgrounds, there were no significant differences in the mean number of total coliforms or $E$. coli on each surface. These results suggest that the of $E$. coli and total coliforms on these surfaces may not be present as a result of hand contact from the children using these playgrounds. With equal rates of these indicator bacteria on high and low hand contact surfaces, it seems more likely that they are present as a result of some other environmental sources, such as birds or other animals that come in contact with the playgrounds.

These results did demonstrate that 12 out of the 60 samples were in fact positive for E. coli. This by itself is a significant result, as $E$. coli are indicators of fecal contamination, and thus demonstrate the potential for the presence of human pathogens from fecal sources (Montville, 2008). The Public Health Agency of Canada (2015), states that the infectious dose for enterohemorragic $E$. coli may be as little as 10 vegetative cells. 
As there were no previous studies present in literature regarding the presence of E. coli or total coliforms specifically on elementary school playground surfaces, further research should be conducted to demonstrate if these results are typical of school playground equipment.

However, these results can be compared to previous research regarding the presence of bacteria in the daycare setting. Daycares typically include children under the age of 5, while elementary schools include children from ages 5 up to 11 or 13 . However, they are similar in that they have high numbers of children in close contact on a daily basis, and many daycares and schools share similar playground equipment. Daycares, like schools are common for their association with disease outbreaks among children. Ibfelt et al (2015) found only a few daycare surfaces to be positive for $E$. coli. However, they noted that this contrasted the results of many previous studies which found $20 \%$ to $50 \%$ of toys, sinks and tables to be positive for fecal coliforms (Ibfelt et al, 2015). This evidence strongly suggests that daycare equipment such as toys and playgrounds may play a role as fomites for the indirect transmission of pathogens from person to person.

\section{Recommendations}

The question of where these bacteria are coming from is important, as it may guide future research related to this topic as well as the practical application of this study's results. If it should be found that they are primarily from environmental sources, such as birds and animals, then this may support the need for disinfecting playground surfaces on a regular basis. Fecal contamination from birds as a source of pathogens was supported by French et al (2009). This study investigated the prevalence of Campylobacter jejuni in bird feces found on playground surfaces in the United Kingdom and New Zealand (French et al, 2009). They demonstrated that $12.5 \%$ of the bird droppings sampled were positive for $C$. jejuni and that the strains present in these samples were identical to those isolated from humans (French et al, 2009). For this reason, they suggest that fecal contamination on playgrounds should be considered as a possible contributor to campylobateriosis in preschool children (French et al, 2009). Given the number of surfaces positive for $E$. coli in this study, it would be advisable to investigate their source. If mostly of bird origin, then it would be worthwhile conducting a similar study to French et al (2009) to see if these surfaces might be positive for $C$. jejuni.

An example of animal fecal contamination of playgrounds resulting in illness can be observed in a study by Staff et al (2012). This study traced the cause of a salmonella outbreak amongst children to a playground contaminated with fecal matter from local wildlife (Stafe et al, 2012). Staff et al (2012) noted that the Salmonella appeared to be able to survive in playground sand for up to 9 months.

Alternatively, evidence suggesting that this E. coli contamination originated from students would be more consistent with studies by Lee and Greig (2010). A study of outbreaks in schools done by Lee and Greig (2010), suggests that students are frequently the source of the pathogens involved in the outbreak, while disease transmission may occur through indirect contact via fomites. Lee and Greig (2010) identified the most common measures for outbreak control in schools involved alerting the health authority, treating the ill and increasing hand washing and use of hand sanitizers. 
Regardless of their origin, the presence of $E$. coli on playground surfaces observed in this study emphasizes the importance of hand washing in the school setting. A study in Pensylvania revealed that $71 \%$ of $7^{\text {th }}$ grade students seldom washed their hands before eating in the school cafeteria (Haapala and Probart, 2004). Students should be educated about the importance of hand washing at an early age in order to develop good habits. School staff should also consider regular hand washing routines for students coming off of playground equipment, particularly if they will be eating shortly afterwards.

The results of this study and others suggests that Environmental Health Officers should inspect school on a regular basis and pay attention to playground sanitation and hand washing protocols. EHO's may be able to educate staff and students about the importance of hand hygiene and sanitation protocol in preventing the spread of disease. If schools do not have an outbreak plan, EHO's would also be a valuable resource in its development.

Finally, in addition to hand washing, consideration should be given to disinfecting playground surfaces on a regular basis. Enserink et al (2015), found that disinfection of fomites was protective with regard to disease outbreak rates in daycares. While we can not assume that this would be true for elementary schools, it would be a prudent precaution none the less.

\section{Limitations}

It must be conceded that this study had a significant number of limitations, particularly with regards to its duration, sample size and budget. This study was limited to sampling surfaces at 15 elementary schools in Abbotsford, BC.
Ideally, sampling 30 or more schools would be recommended in order to account for variations between schools.

A significant source of error in this study might have been the inability to sample identical types of surfaces at each school. For instance, the building materials for playgrounds varied from school to school, and sampled surfaces, while similar in function, varied in their composition from bare metal, to painted metals and plastic. Viruses and bacteria are known to have significantly different survival times on different materials. This was demonstrated for norovirus by Kim et al (2014), who showed that norovirus remained viable on wood surfaces for much longer than other surfaces such as steel. Similarly, Wilks et al (2005) demonstrated that E. coli 0157 numbers dropped drastically after just 2 hours on copper and nickel surfaces, while viable cells were recovered from stainless steel for up to 28 days.

In order to maintain consistent sampling conditions, all 60 samples were taken on the same day. It is conceivable, however, that sampling during different weather conditions or at different times of the year might yield different results. For instance, the playground may receive more use in spring and summer months as compared to fall or winter.

One final limitation in methodology was the timing of swabbing vs plating. Due to the limited access to school grounds, sampling was conducted from 4:00pm to 8:00pm, while the plating of these samples was not conducted until 2:30pm the next day. According to the 3M Quick Swab manufacturer instructions, this method is acceptable if the swab samples are kept cold, as they were in this study. However, there may be some potential for the loss of 
a few stressed cells during this refrigeration period prior to plating.

\section{Future Research Suggestions}

The results of this study bring into question the sanitation and thus safety of elementary school playgrounds. Further research should be done to see if these results are typical of $E$. coli levels on other types of playground surfaces. If they are, it would be useful to know what the origins of these $E$. coli might be. If they are coming from fecal contamination from animals that come into contact with the playground, then investigating the presence of other pathogens would tell us about the actual risk for disease transmission to children using this equipment.

Sampling of total aerobic counts could be conducted on the surfaces sampled in this study to see if there are any differences in the numbers of bacteria on these surfaces as opposed to just $E$. coli or total coliforms. This would provide evidence that playgrounds could still be reservoirs for bacteria deposited by the hands of school children.

Other microbes of interest on school playgrounds would include viruses. In particular, Norovirus is known to be transmitted via the fecal-oral route, just like pathogenic $E$. coli strains (Goodgame, 2006), and can survive for over 28 days on stainless steel surfaces (Kim et al, 2014). This would certainly justify the disinfection of playground surfaces in the event of a gastrointestinal outbreak.

Further research might also be done regarding the rates and quality of hand washing by elementary school children. For example, how many school staff have their students wash their hands prior to eating lunch or after coming off the playground? Do school children understand how to wash their hands properly, and at what age can they be trusted to do this without supervision? Research has been done on this topic in the U.S. and the U.K. (Haapala and Probart, 2004), though current knowledge for $\mathrm{BC}$ schools would be beneficial.

\section{Conclusion}

The purpose of this study was to investigate the possibility of playgrounds acting as a temporary reservoir for pathogens deposited by the hands of children. This results of this study did not support its hypotheses, and thus we cannot conclude that the number of total coliforms or E. coli are significantly different on high or low hand contact surfaces on elementary school playgrounds. However, it did provide evidence that a significant number of playground surfaces may be positive for $E$. coli, indicating the presence of fecal contamination on playground surfaces.

\section{Acknowledgments}

The authour would like to thank Fred Shaw for managing supply orders and making his lab available for this project, Vanessa Karakilic and Helen Heacock for their guidance on project topics and timelines, and Sarah Fowler for escorting the author on school properties.

\section{Competing Interests}

The authors declare that they have no competing interests. 


\section{References}

3M. (2010). 3M Petri film Plates: MPN

Comparison Sheet. Retrieved from

http://solutions.3m.com/wps/portal/3M/en

US/Microbiology/FoodSafety/product-

information/product-

catalog/?PC Z7 RJH9U523003DC023S7P92

O3087000000 nid=COWJ62882Vbe29BDXSB

$\underline{\mathrm{JFgl}}$

3M. (2011). 3M Petrifilm ${ }^{\mathrm{TM}}$ E. coli/Coliform

Count Plate: Product Instructions. Retrieved from

http://multimedia.3m.com/mws/media/701

9510/product-instructions-3m-petrifilm-ecoli-coliform-count-plate.pdf

3M. (2014). 3M Petrifilm ${ }^{\mathrm{TM}}$ E. coli/Coliform

Count Plate: Interpretation Guide. Retrieved from

http://solutions.3m.com/wps/portal/3M/en US/Microbiology/FoodSafety/productinfor mation/productcatalog/?PC Z7 RJH9U5230 03DC023S7P9203087000000 nid=COWJ628 82Vbe29BDXSBJ7FgI

3M. (2014). 3M Quick Swab: Product Instructions. Retrieved from http://solutions.3m.com/wps/portal/3M/en US/Microbiology/FoodSafety/productinformation/productcatalog/?PC Z7 RJH9U523003DC023S7P92 O3087000000 nid=P1K9V2S1X9beZD53Z9P $\underline{\mathrm{HFHgl}}$

BC Centre for Disease Control. (2014). Environmental testing. Retrieved from http://www.bccdc.ca/PHSALaboratories/Lab oratoryTestsandRequisitionForms/Environm entalTesting.htm
BD. (2012). BD Prepared Environmental Monitoring Plates. Package Insert. Sparks, MD, USA: Becton, Dickinson and Company. Retrieved from https://www.bd.com/ds/technicalCenter/ins erts/8817741(1006).pdf

Blackburn, C. W., Baylis, C. L., \& Petitt, S. B. (1996). Letters in applied microbiology: Evaluation of petrifilm methods for enumeration of aerobic flora and coliforms in a wide range of foods Blackwell Publishing.

Chollet, R., \& Ribault, S. (2012). Use of ATP bioluminescence for rapid detection and enumeration of contaminants: The milliflex rapid microbiology detection and enumeration system. Rijeka, Croatia: InTech.

Claro, T., Galvin, S., Cahill, O., \& FitzgeraldHughes, D. (2007). What is the best method? recovery of methicillin-resistant staphylococcus aureus and extendedspectrum $\beta$-Lactamase-Producing escherichia coli from inanimate hospital surfaces. Infection Control and Hospital Epidemiology, 35(7), 869; 869-871; 871.

Enserink, R., Mughini-Gras, L., Duizer, E. \& Kortbeek, T. (2010). Risk factors for gastroenteritis in child day care. Epidemiology and Infection, 143(13), 2707; 2707-2720; 2720.

French, N.P., Midwinter, A., Holland, B., Collins-Emmerson, J., Pattison, R., Colles, F., and Carter, P. (2009). Molecular Epidemiology of Campylobacter jejuni Isolates from Wild-Bird Fecal Material in Children's Playgrounds. Applied and Environmental Microbiology, 75(3), 779-783. 
Goodgame, R. (2006). Norovirus gastroenteritis. Current Gastroenterology Reports, 8(5), 401-408. doi:10.1007/s11894006-0026-4.

Greig, J. D., \& Lee, M. B. (2008). A review of enteric outbreaks in child care centers: Effective infection control recommendations. Journal of Environmental Health, 71, 24+.

Heacock, H. \& Karakilic, V. (2015). Module 5: Inferential Statistics. [Lecture Slides]. BCIT ENVH 8400 - Research Methods, Burnaby, BC.

Haapala and Probart. (2004). Food safety knowledge, perceptions, and behaviors among middle school students. Journal of Nutrition Education and Behavior, 36(2), 7176.

Ibfelt T, Engelund EH, Permin A, Madsen JS, Schultz AC, Andersen LP. (2015). Presence of pathogenic bacteria and viruses in the daycare environment. J Environ Health 10(78), 24.

Jasson V, Jacxsens L, Luning P, Rajkovic A. (2010) Alternative microbial methods: An overview and selection criteria. Food Microbiol, 27(6):710; 710-730; 730.

Jordano, R., Lopez, C., Rodriguez, V., Cordoba, G., Medina, L. M., \& Barrios, J. (1995). Comparison of petrifilm method to conventional methods for enumerating aerobic bacteria, coliforms, escherichia coli and yeasts and molds in foods. Acta Microbiologica Et Immunologica Hungarica, 42(3), 255.
Kim, A., Park, S., Bae, S., Oh, M., \& Ha, S. (2014). Survival of norovirus surrogate on various food-contact surfaces. Food and Environmental Virology, 6(3), 182-188. doi:10.1007/s12560-014-9154-4

Lee, M., Marilyn B Lee, \& Judy D Greig. (2012). A review of gastrointestinal outbreaks in schools: Effective infection control interventions. The Journal of School Health, $80(12), 588 ; 588$.

Montville, T. J. (2008). Food microbiology: An introduction ASM Press.

Mulvey, D., Mulvey, D., Redding, P., Robertson, C., \& Woodall, C. (2011). Finding a benchmark for monitoring hospital cleanliness. The Journal of Hospital Infection, 77(1), 25; 25-30; 30.

Olivier, R. (2013). Comparison of Aerobic and E. Coli Colony-Forming Units Isolated From Circulating Paper and Plastic \$20 Canadian Banknotes. Environmental Health Journal. Retrieved from http://www.ncceh.ca/sites/default/files/BCl T-Olivier-2013.pdf

Public Health Agency of Canada. (2014). Norovirus - pathogen safety data sheet. Retrieved from http://www.phacaspc.gc.ca/lab-bio/res/psds-ftss/msds112eeng.php

Public Health Agency of Canada. (2015). Escherichia coli, enterohemorrhagic. Retrieved from http://www.phacaspc.gc.ca/lab-bio/res/psds-ftss/msds63eeng.php 
Saab khudhir, Z., Hadi Najim, N., \& H.Yousif, D. (2013). Comparison between the Petrifilm ${ }^{\mathrm{TM}}$ and the conventional methods for enumerating aerobic bacteria and $E$. coli in locally produced soft cheese in baghdad. Bas.j. Vet.Res., 12(2), 203-203 - 211.

SAS University Edition [Computer Software]. (2015). Retrieved from http://www.sas.com/en ca/software/univer sity-edition.html

Staff, M., Musto, J., Hogg, G., Janssen, M., and Rose, K. (2012). Salmonellosis outbreak traced to playground sand, Australia, 20072009. Emerging Infectious Disease, 18(7), 1159-1162.

Vlosky, R.P., and Shupe, T.F. (2005).

Manufacturer's perceptions about using treated wood in children's playground equipment. Forest Products Journal, 55(12), 190-193.

Wagner, J., \& Clodfelter, S. (2014). Preventing diseases and outbreaks at child care centers using an education, evaluation, and inspection method. Journal of Environmental Health, 76, 18+.

Wilks, S. A., Wilks, S. A., Michels, H., \& Keevil, C. W. (2005). The survival of escherichia coli O157 on a range of metal surfaces. International Journal of Food Microbiology, 105(3), 445; 445-454; 454. 